\title{
Role of Pharmacists in Pharmacogenomics
}

\author{
Gamal Osman Elhassan*, Sana Hashmi, Syedda Ayesha Farhana, Kamal A Qureshi and Riyaz A Khan \\ Department of Pharmaceutics, Unaizah College of Pharmacy, Qassim University, Unaizah, Kingdom of Saudi Arabia
}

Pharmacists' roles in pharmacogenetics have remained as poorly defined, and have a little or negligible role to play in their own field and is expected to continue like this in the future as well. However, in actual clinical set up, practitioners whose training and practices involve clinical monitoring of drug treatment, pharmacists are in a valuable position to help them define the role of pharmacogenetics in the eventual outcome of the pharmacotherapy.

Pharmacogenomics refers to the use of the genetic information of patients or diseased tissues (e.g. cancer tissue of a patient) to aid prescribers in selecting the correct drug and dose for the patient. Genetic data can reveal a patient's risk for adverse effects or toxicity from a particular drug or the likelihood that the drug will have any effect at all [1].

The field of pharmacogenetics provides use with diverse opportunities for pharmacists in helping healthcare workers in optimizing therapeutic outcome of intended pharmacotherapy. Pharmacists' role in pharmacogenetics falls within following three major domains [2]:

- Establishment of tests involving pharmacogenetics in clinical practices.

- Development of newer methodologies for research and providing research directions.

- Educating healthcare providers and helping in the development of infrastructure and create technologies for pharmacogenetics towards its actual implementation in health care system [3].

\section{Establishment of Test Involving Pharmacogenetics in the Clinical Practice}

Pharmacists play an important role in implementation of pharmacogenetics services in clinical setting by their services at the point of prescription, at the point of dispensing and integration of new pharmacogenetics services.

\section{Pharmacogenetics at the point of prescription}

At present, pharmacogenetics at the point of prescribing is likely to involve only a small number of pharmacists in highly specialized roles, such as in oncology pharmacy, although this is likely to change in the future, permeating through into the primary care setting. The persons most likely to order a test at this point in the care pathway are the prescribers themselves, using the information obtained to inform drug choice and dosage. As pharmacists increasingly take on prescribing roles, this aspect of pharmacogenetics is likely to increase in importance and relevance.

\section{Optimizing drug therapy}

Pharmacists are expected to take center stage in the safe and ethical use of pharmacogenetic tool to optimize the drug therapy.
With the help of Pharmacogenetics, pharmacists help analyze how an individual's genetic makeup interacts with a prescribed drug and also help in providing information on individual's genetic influence on the body's response to medications pharmacologically.

\section{Pharmacogenetics at the point of dispensing}

Hospital pharmacists may play a greater role in the application of some of these emerging therapies, but this is unlikely to have a major impact on demand. Within the current practice framework, pharmacogenetics advice, based on information from various sources, notably the drug data sheets, can be given by the pharmacist at the point of dispensing and sale. The advisory role of the pharmacist, which includes informing patients about how to take their medications and how to avoid drug-drug and drug-diet interactions, is simply expanded to include pharmacogenetics without structural alteration in practice pathways.

\section{Integration of pharmacogenetics services}

This aspect will require alteration in clinical practice but will build on the expanded services, which are being developed or piloted by pharmacists with the support of the Department of Health. Examples include the provision of testing for Chlamydia, diabetes and blood pressure monitoring, and medicines management services. Drug monitoring, such as of warfarin, with blood coagulation testing (prothrombin INR measurement) which takes account of cytochrome P450 2C9 and VKORC1 genotypes polymorphism, may well form part of this service.

Some specialty areas in pharmacy profession have been recognized where pharmacist play important role such as:

\section{Pharmacogenetics within oncology pharmacy}

Within the oncology setting, pharmacogenetics is a part of existing pharmacy practice and may well be the area where uptake of pharmacogenetics will be greatest in the short-term. Experience gained in this setting should be collated and disseminated to other clinical areas to aid the integration of pharmacogenetics as it becomes clinically appropriate. In cancer treatment, genetic testing can pinpoint patients

*Corresponding author: Gamal Osman Elhassan, Unaizah College of Pharmacy Qassim University, Unaizah 51452, Qassim, Kingdom of Saudi Arabia, Tel: 00966530965955, 00966543080845; E-mail: go.osman@qu.edu.sa

Received: June 16, 2017; Accepted June 26, 2017; Published June 29, 2017

Citation: Elhassan GO, Hashmi S, Farhana SA, Qureshi KA, Khan RA (2017) Role of Pharmacists in Pharmacogenomics. J Pharmacovigil 5: e170. doi:10.4172/2329$6887.1000 \mathrm{e} 170$

Copyright: (c) 2017 Elhassan GO, et al. This is an open-access article distributed under the terms of the Creative Commons Attribution License, which permits unrestricted use, distribution, and reproduction in any medium, provided the original author and source are credited. 
who will benefit from certain drug therapies. For a woman with breast cancer, a diagnostic test can determine her tumor's genetic signature. If the patient tests positive for human epidermal growth factor receptor 2 (HER-2), she can be given trastuzumab, which suppresses excess HER2 and thereby $50 \%$ reduction in the recurrence risk of disease [4].

\section{Developing Methodologies for Research and Providing Research Directions}

Pharmacist's role with regard to the pharmacogenetics is expected to be patient oriented and targeted to optimize the disease and healthrelated quality of life outcomes. However, pharmacists are also expected to play crucial role in driving the early stages of clinical and translational pharmacogenetic research by dint of their background of a drug's pharmacokinetics, pharmacodynamics, and the pharmacoeconomics that help maximize health care delivery by acting either principal or co-investigator or a key member of a research team. In both academia and pharmaceutical industries alike, pharmacists are involved from beginning to the end of a research involving pharmacogenomics to study specific diseases in wherein drug therapy is an integral component of a disease management. In particular, at places where the focus is on to target pharmacotherapies to which patients have become nonresponsive or intended pharmacotherapy has become ineffective in some patient population group and is effective in only some of patient population group (i.e., large heterogeneity in clinical response) and also are attributed to cause adverse effects.

It is well known that single gene polymorphism greatly influence response of a drug on drug (e.g., influence of thiopurinemethyl transferase polymorphisms on azathioprine-induced myelo suppression), however, most drug responses are very intricate and involve multiple genes which constitute a drug response "pathway." Therefore, unfolding various genes involved in drug responses involving such drug-response pathways could represent a useful tool in the selection/choice of drugs for diseases at the early outset of the research involving pharmacogenomics. Polymorphisms of the genes involved in a particular drug response pathway could adversely influence clinical response of drugs e.g. pharmacological response of phenytoin. However, there is a limitation to this approach, as understanding of all the relevant components (genes and proteins) of a drug pathway is less well understood till date. It must be discerned that pharmacist are an expert in pharmacotherapy together with pharmacokinetic and pharmacodynamics components of a clinically prescribed drug (e.g. metabolizing enzymes, transporters, drug targets). Hence, the pharmacist must be seen as, integral part in the construction of comprehensive pathways for better treatment outcomes involving pharmacogenomics [5].

As illustrated above, pharmacists possess expertise and competency in areas that are highly relevant and could impart valuable inputs from the very outset till the final stages of pharmacogenomics research.

\section{Participating in Education and Infrastructure Development that Moves Pharmacogenetics Technologies toward Implementation}

\section{Pharmacogenetics with community pharmacy}

Some participants from the community pharmacy setting voiced the opinion that there would need to be drivers to integrate pharmacogenetics into practice, both in terms of clinical evidence and financial incentives. It was also felt that the education and information for community pharmacists would need to be at a very practical level; i.e., 'this drug should not be prescribed unless this test has been carried out.

\section{Pharmacogenetics within drug development and regulation}

Participants believed that as pharmacogenetics becomes embedded into the drug development process this would lead to the development of genetic tests to accompany an increasing number of new drugs. Pharmacists working in drug-regulatory bodies and the Department of Health have an important role to play in facilitating an evaluation system and championing the uptake of drug-test combinations that fulfill the relevant clinical and economic criteria. This section will underline further consideration to the nature of the tasks that this role would require and the skills that pharmacists would need to fulfil the role. It has been agreed upon during the meeting from the NHS National Genetics Education and Development Centre that there is a need to carry out an exercise with participants to identify the skills required by pharmacists who could help integrate pharmacogenetics into their practice [6].

\section{References}

1. Streetman DS (2007) Emergence and evolution of pharmacogenetics and pharmacogenomics in clinical pharmacy over the past 40 years. Ann Pharmacother 41: 2038-2041.

2. Brock TP, Valgus JM, Smith SR, Summers KM (2003) Pharmacogenomics: implications and considerations for pharmacists. Pharmacogenomics 4: 321-330.

3. Ellis SL, Carter BL, Malone DC, Billups SJ, Okano GJ, et al. (2000) Clinical and economic impact of ambulatory care clinical pharmacists in management of dyslipidemia in older adults: the improve study: Impact of Managed Pharmaceutical Care on Resource Utilization and Outcomes in Veterans Affairs Medical Centers. Pharmacotherapy 20: 1508-1516.

4. LeeW, LockhartAC, Kim RB, Rothenberg ML (2005)Cancerpharmacogenomics: powerful tools in cancer chemotherapy and drug development. Oncologist 10 104-111.

5. Brock TP, Faulkner CM, Williams DM, Smith SR (2002) Continuing education programs in pharmacogenomics for pharmacists. Am J Health Syst Pharm 59: 722-725.

6. El-lbiary SY, Christine C, Brian A (2008) Potential roles for pharmacists in pharmacogenetics. Amer Pharma Asso 48: e21-e32. 